\title{
Masked Presentations of Emotional Facial Expressions Modulate Amygdala Activity without Explicit Knowledge
}

\author{
Paul J. Whalen, Scott L. Rauch, Nancy L. Etcoff, Sean C. Mclnerney, Michael B. Lee, and Michael A. Jenike \\ Psychiatric Neuroimaging Research Group and Nuclear Magnetic Resonance Center, Massachusetts General Hospital \\ and Harvard Medical School, Boston, MA 02115
}

Functional magnetic resonance imaging (fMRI) of the human brain was used to study whether the amygdala is activated in response to emotional stimuli, even in the absence of explicit knowledge that such stimuli were presented. Pictures of human faces bearing fearful or happy expressions were presented to 10 normal, healthy subjects by using a backward masking procedure that resulted in 8 of 10 subjects reporting that they had not seen these facial expressions. The backward masking procedure consisted of $33 \mathrm{msec}$ presentations of fearful or happy facial expressions, their offset coincident with the onset of $167 \mathrm{msec}$ presentations of neutral facial expressions. Although subjects reported seeing only neutral faces, blood oxygen level-dependent (BOLD) fMRI signal in the amygdala was significantly higher during viewing of masked fearful faces than during the viewing of masked happy faces. This difference was composed of significant signal increases in the amygdala to masked fearful faces as well as significant signal decreases to masked happy faces, consistent with the notion that the level of amygdala activation is affected differentially by the emotional valence of external stimuli. In addition, these facial expressions activated the sublenticular substantia innominata (SI), where signal increases were observed to both fearful and happy faces-suggesting a spatial dissociation of territories that respond to emotional valence versus salience or arousal value. This study, using $\mathrm{fMRI}$ in conjunction with masked stimulus presentations, represents an initial step toward determining the role of the amygdala in nonconscious processing.

Key words: amygdala; extended amygdala; substantia innominata; nucleus basalis of Meynert; bed nucleus of the stria terminalis; emotion; facial expression; backward masking; awareness; fMRl; neuroimaging
Neuroscientific research provides many examples of tasks executed below the level of awareness (Schacter et al., 1993; He et al., 1996; Berns et al., 1997; Rauch et al., 1997). It has been argued that initial responses to affective stimuli are automatic and do not require awareness (Zajonc, 1980). The amygdala is a brain area located within the medial temporal lobe that is known to process affective or emotionally valenced stimuli (see Aggleton, 1992). On the basis, in part, of animal studies demonstrating a direct short-latency pathway from the thalamus to the amygdala (LeDoux et al., 1985), LeDoux (1996) has proposed that the amygdala might survey emotionally valenced stimuli without awareness. Consistent with this notion, studies of human subjects by Öhman and colleagues (Öhman, 1992) have demonstrated skin conductance responses to emotionally valenced facial expressions conditioned to predict an aversive electrical shock when these expressions were presented in a manner that prevented awareness (i.e., backward masking). Taking a lead from human lesion data (Adolphs et al., 1995; Calder et al., 1996), recent neuroimaging reports in humans have demonstrated activation within the amyg-

Received Aug. 28, 1997; revised Oct. 13, 1997; accepted Oct. 20, 1997.

This work was supported by a Harvard Medical School Clinical Research Training Program (CRTP) Fellowship to P.J.W. (MH-16259; Robert McCarley and Stuart Hauser, Program Directors), MH-01215 and National Alliance for Research on Schizophrenia and Depression Award to S.L.R., and the David A. Judah Fund to M.A.J. We thank Terry Campbell, Mary Foley, Ivan Audouin, Sherry Pai, Aiping Jiang, and George Bush for technical assistance; and Richard McNally, Bruce Kapp, Tim Curran, Daniel Schacter, Robert Savoy, Randy Buckner, Cary Savage, Elizabeth Phelps, and Bruce Rosen for advice and comments.

Correspondence should be addressed to Dr. Paul J. Whalen, Department of Psychiatry, Massachusetts General Hospital, 13th Street, Building 149, CNY-9, Charlestown, MA 02129. E-mail: paulw@nmr.mgh.harvard.edu

Copyright (C) 1997 Society for Neuroscience $0270-6474 / 97 / 180411-08 \$ 05.00 / 0$ dala in response to facial expressions of emotion (Breiter et al., 1996; Morris et al., 1996). The present study used functional magnetic resonance imaging (fMRI) during the presentation of backwardly masked facial expressions to determine whether amygdala activation might be demonstrated in humans in the absence of explicit knowledge.

A backward masking procedure previously demonstrated to interrupt processing of emotionally expressive faces (Esteves and Öhman, 1993; Rolls and Tovee, 1994) was used. Each masked stimulus consisted of a $33 \mathrm{msec}$ fearful or happy expression face (target), its offset coincident with the onset of a $167 \mathrm{msec}$ neutral expression face (mask). Esteves and Öhman (1993) demonstrated that if the stimulus onset asynchrony (SOA; i.e., the interval between the onset of the target and the mask) was sufficiently brief $(<40 \mathrm{msec})$, human subjects were not aware of the emotionally expressive target face, as defined by objective forced choice tasks and subjective report. In the present study 10 human subjects viewed repeating $28 \mathrm{sec}$ epochs that consisted of either fearful faces masked by neutral faces, happy faces masked by neutral faces, or a single cross $(+)$ that served as a fixation point baseline. We predicted that, although subjects would report seeing only the neutral masks, blood oxygen level-dependent (BOLD) fMRI signal intensity (Kwong et al., 1992; Ogawa et al., 1992) would increase in the amygdala in response to masked fearful targets when compared with masked happy targets. In addition, we predicted that presentation of masked emotional facial expressions would serve to isolate amygdala activation in contrast to previous neuroimaging studies of nonmasked facial expressions, which have demonstrated activation of the amygdala along with numerous additional brain regions (Breiter et al., 1996; Morris et al., 1996). 


\section{MATERIALS AND METHODS}

Subjects. Ten right-handed males aged 19-32 (mean, 23.8 years) provided informed consent before participation in this study, according to guidelines established by the Subcommittee on Human Studies at the Massachusetts General Hospital. Handedness was defined by the Edinburgh Inventory (Oldfield, 1971). Subjects were told that they would be presented with pictures of faces. All subjects were naive to the face stimuli used and to our hypotheses pertaining to the emotional expressions of faces. For this initial experiment a single gender cohort was studied to minimize heterogeneity, thereby improving statistical power. Future studies of female subjects will be necessary to determine the generalizability of the current results.

Selection of fear faces as threat-related stimuli. Face stimuli consisted of fearful, happy, and neutral expressions of eight individuals (numbers 021, 030, 040, 081, 101, 121, 131, 140; Ekman and Friesen, 1976). We selected fearful faces as our negatively valenced stimuli because human lesion studies document a deficit in the processing of fearful faces after amygdala lesions (Adolphs et al., 1995; Calder et al., 1996), and previous neuroimaging studies demonstrate amygdala activation to these stimuli (Breiter et al., 1996; Morris et al., 1996). We offer the concept that, unlike an angry face that represents a direct threat, the relationship of a fearful face to threat is ambiguous in that a fearful face signals the presence of danger, but not its source. In this sense, a fearful face can be conceptualized as a contextual stimulus, whereas an angry face can be conceptualized as a specific cue.

Paradigm. Subjects were presented with alternating $28 \mathrm{sec}$ epochs of masked fearful face targets (F), masked happy face targets $(\mathrm{H})$, or a single cross that served as a low-level fixation condition $(+)$. During each epoch subjects viewed either 56 masked fearful stimuli or 56 masked happy stimuli (each of eight fearful or happy faces was masked by the neutral expression for each of the other seven individuals).

Masked stimuli were presented twice per second in a random order. Each $200 \mathrm{msec}$ masked stimulus consisted of a $33 \mathrm{msec}$ fearful or happy expression (target) immediately followed by a $167 \mathrm{msec}$ neutral expression (mask).

The order of $28 \mathrm{sec}$ epochs containing 56 fearful or happy masked stimuli was counterbalanced within and across subjects; one-half of the subjects viewed masked fearful, followed by masked happy, targets during their first run $(+, \mathrm{F}, \mathrm{H},+, \mathrm{F}, \mathrm{H},+, \mathrm{F}, \mathrm{H},+)$; the other half viewed masked happy, followed by masked fearful, targets during their first run $(+, \mathrm{H}, \mathrm{F},+, \mathrm{H}, \mathrm{F},+, \mathrm{H}, \mathrm{F},+)$. Then the order of fearful and happy target epochs was reversed for the second run for all subjects. These 10 epochs comprised a $4 \mathrm{~min}$ and $40 \mathrm{sec}$ run. Each subject viewed two runs.

Subject debriefing. Subjective report measures were used to assess the subjects' explicit knowledge of presented masked facial expressions of emotion after the completion of all stimulus presentations. Immediately after the experiment the subjects were asked to describe any aspect of the presented faces. Next, the subjects were asked to comment on the emotional expressions of the faces. Then the subjects were asked if they had seen any happy or smiling faces and asked if they had seen any fearful or afraid faces. Finally, the subjects were shown all face stimuli (fearful, happy, and neutral) and asked to point out the specific faces they had referred to in response to earlier questions.

Stimuli and apparatus. Face stimuli consisted of PICT files that were assembled frame by frame into a film, using Adobe Premiere software. Specialized hardware (Media 100, Marlboro, MA) was used to transfer the digital PICT information to videotape synchronized with the headsweeps of the VCR so as not to distort the stimuli. A VCR was used to play the tape, and the output was projected (Sharp XG-2000U LCD) onto a screen within the imaging chamber, viewable by a mirror $(1.5 \times 3.5$ inches) $\sim 6.5$ inches from the subject's face. The play speed of the VCR was 30 frames $/ \mathrm{sec}$, creating a $33 \mathrm{msec} /$ frame presentation rate.

Pulse rate was measured from the right index finger of all subjects during stimulus presentations via pulse oximetry (In Vivo Systems, Orlando, FL).

Functional magnetic resonance images were collected in a General Electric Signa 1.5 Tesla high-speed imaging device (modified by Advanced NMR Systems, Wilmington, MA), using a quadrature head coil. Our Instascan software is a variant of the echo planar technique first described by Mansfield (1977). Head stabilization was achieved with a plastic bite bar molded to each subject's dentition.

Image acquisition and data analysis. Our standard image acquisition protocol was used and previously has been detailed elsewhere (Cohen and Weisskoff, 1991; Kwong, 1995). An initial sagittal localizer [spoiled gradient recall acquisition in a steady state (SPGR), 60 slices, resolution
$0.898 \times 0.898 \times 2.8 \mathrm{~mm}$ ] was performed to provide a reference for future slice selection and for eventual localization within Talairach space (Talairach and Tournoux, 1988). After automated shimming (Reese et al., 1995) to maximize field homogeneity, a magnetic resonance (MR) angiogram (SPGR, resolution $0.78125 \times 0.78125 \times 2.8 \mathrm{~mm}$ ) was acquired to identify large- and medium-diameter vessels. Then a set of T1weighted high-resolution transaxial anatomic scans (resolution $3.125 \times$ $3.125 \times 8 \mathrm{~mm}$ ) was acquired. For the functional series, asymmetric spin echo (ASE) sequences were used to minimize macrovascular signal contributions. Functional ASE data were acquired as 15 contiguous, interleaved, horizontal $8 \mathrm{~mm}$ slices that paralleled the intercommissural plane (voxel size $3.125 \times 3.125 \times 8 \mathrm{~mm}$; 100 images per slice, TR/TE/ Flip $\left.=2800 \mathrm{msec} / 70 \mathrm{msec} / 90^{\circ}\right)$.

Automated data analytic techniques began with a quantification of subject motion and then correction, using an algorithm developed by Jiang et al. (1995), based on Woods et al. (1992). Both functional and high-resolution structural data were placed into normalized Talairach space and resliced into $3.125 \times 3.125 \times 3 \mathrm{~mm}$ voxels in the coronal plane. Then data from individuals were baseline-normalized and concatenated (averaged). Nonparametric statistical maps were calculated with the Kolmogorov-Smirnov (KS) statistic, displayed in pseudocolor, scaled according to significance, and superimposed on T1-weighted highresolution images also placed into Talairach space and resliced in the coronal plane. Because we predicted only amygdala activation to the present experimental manipulation, our a priori significance threshold $\left(p<6.6 \times 10^{-4}\right)$ represents a Bonferroni-corrected 0.05 probability level based on the $\sim 76$ voxels that make up the amygdaloid region (Filipek et al., 1994).

\section{RESULTS}

\section{Subject debriefing}

Immediately after the experiment, the subjects were asked to describe any aspect of the presented faces. Two of the 10 subjects offered descriptions indicating that they had seen features of the emotional target stimuli. The remaining eight subjects were asked next to comment on the emotional expressions of the faces, and all responded with reference to the neutral mask stimuli alone. Then the subjects were asked if they had seen any happy or fearful faces. All eight subjects reported that they had not seen these expressions. Finally, these eight subjects were shown all face stimuli (fearful, happy, and neutral) and asked to point out the specific faces they had seen. Subjects selected only neutral faces. Therefore, we present here brain activation data for the eight subjects who reported having seen only the neutral faces.

\section{Pulse rate data}

Pulse rate was measured in the eight subjects who reported not having seen the masked fearful and happy faces (sampled approximately every $5 \mathrm{sec}$ ). Technical problems prevented measurement within the magnet for two of these subjects. Results revealed no significant pulse rate changes to the presentation of fearful or happy faces when compared with one another or the fixation baseline condition $(p>0.05)$.

\section{fMRI data}

Figure 1 presents BOLD signal changes across whole brain in response to masked fearful faces versus masked happy faces for the eight subjects who demonstrated no explicit knowledge of the presence of these stimuli. Note the relative absence of activation outside the amygdaloid region.

Figure $2 A$ presents the most posterior coronal slice from the amygdaloid region of activation depicted in Figure 1. Significantly higher BOLD signal is observed in the amygdala in response to masked fearful faces $(467.56 \pm 0.41$, mean \pm SEM) when compared with masked happy faces (464.85 \pm 0.43 , mean \pm SEM). Four contiguous voxels within the right amygdala met the threshold for statistical significance $\left(p<6.6 \times 10^{-4}\right.$; see Materials and 


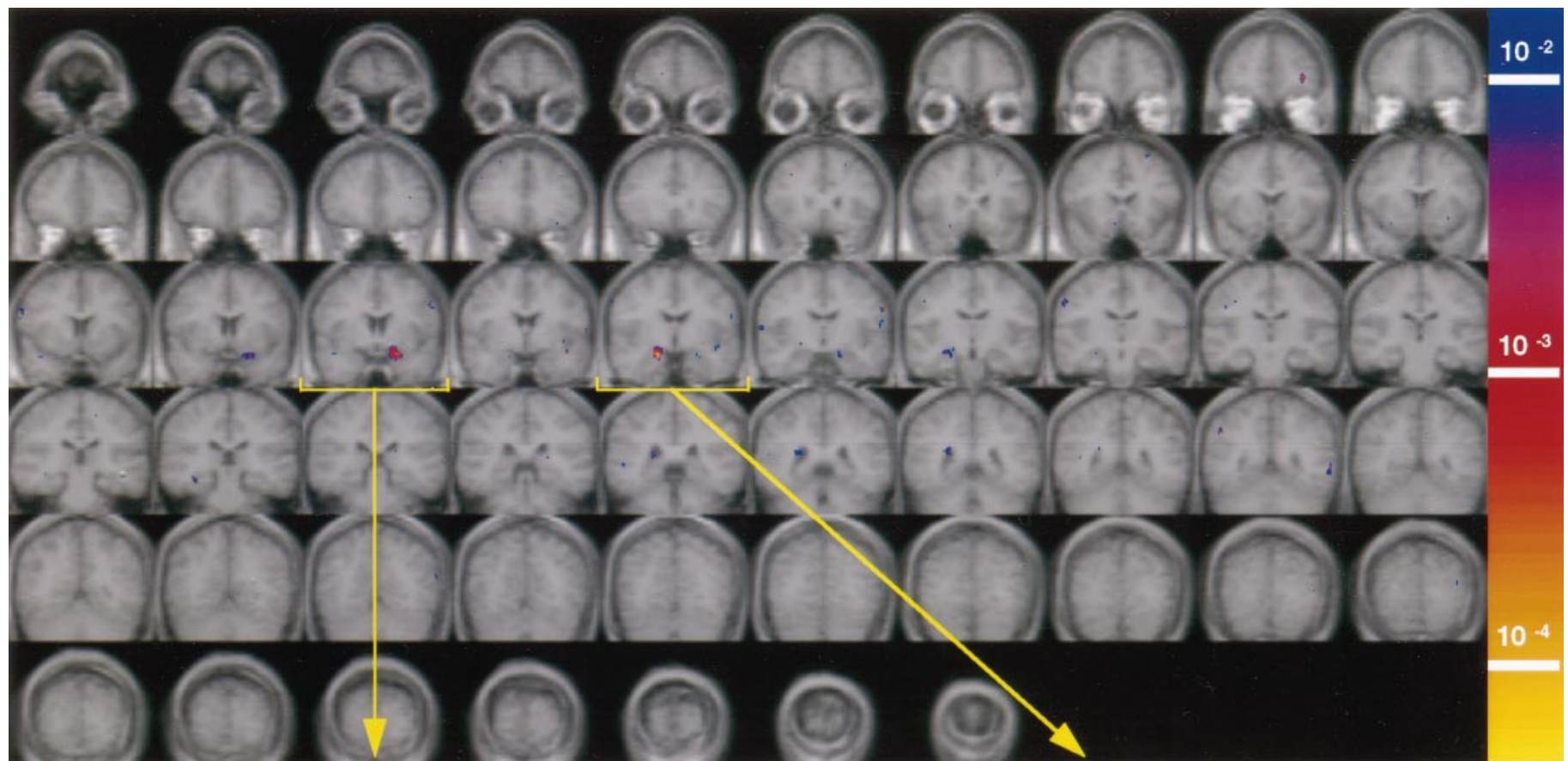

\section{Left Amygdala/SI (y = 0; see Figure 3)}

Right Amygdala (y = -6; see Figure 2)

Figure 1. Masked fearful faces versus masked happy faces. Areas of significant activation $\left(p<6.6 \times 10^{-4}\right)$ across whole brain, presented here as 57 , $3 \mathrm{~mm}$ coronal slices for the masked fear versus masked happy contrast, for the eight subjects who reported not having seen the fearful or happy target faces. All figures are displayed according to radiological convention (i.e., left = right; right = left; top $=$ superior; bottom $=$ inferior). The most anterior slice is in the top left corner, and slices proceed in a posterior direction from left to right and then down. The colorized statistical map is superimposed over the averaged high-resolution structural data for these eight subjects. Both functional and structural data have been placed in a normalized space according to the coordinate system of Talairach and Tournoux (1988). All figures were smoothed by using a Hamming nine voxel 1:2:1 kernel filter, although activations were significant on unsmoothed maps. Significant activation within the amygdaloid region is evident within two slices in the third row (yellow brackets). These two slices represent Talairach coordinates in the $y$-plane of 0 (see Fig. 3) and -6 (see Fig. 2), respectively. Note also the relative lack of activation across all other brain regions. There is an activation of the inferior prefrontal cortex (first row, slice nine) that met the significance level set a priori for the amygdala. Note that, although our 15 original horizontal slice acquisitions covered "whole brain," susceptibility from the sinus space causes signal dropout in portions of some brain regions (see Results).

Methods). We then assessed the direction of signal change to fearful or happy faces in comparison to the fixation baseline epochs. We considered only the four significantly activated voxels from the fear versus happy contrast depicted in Figure $2 A$; the masked fear versus fixation contrast revealed a significant increase in signal intensity, whereas the masked happy versus fixation contrast revealed a significant decrease in signal intensity. For this comparison we treated the four voxels in the amygdala (defined by the overall fear vs happy contrast) as one region of interest (ROI). Mean signal intensity within this ROI demonstrated a significant increase to masked fearful faces and a significant decrease to masked happy faces when compared with fixation $(p<0.05)$. Thus, the larger overall fear versus happy statistical effect $\left(p<6.6 \times 10^{-4}\right)$ demonstrated across these four voxels depends on both a response to the fearful faces (increase) and the happy faces (decrease). By considering only voxels activated in the fear versus happy contrast, we are assured of describing only the nature of signal changes specifically attributable to the emotional expressions.

To delineate the effect of repeated presentations of these stimuli, Figure $2 B$ presents BOLD signal changes in the amygdala for the significant ROI depicted in Figure $2 A$ across all epochs of presentation. First, notice that BOLD signal in the amygdala is always higher during presentation of masked fear faces when compared with the contiguous (counterbalanced) epoch of masked happy faces. For this group of eight subjects the average percentage of change in signal intensity between conditions (masked fear vs masked happy) ranged from 0.77 to $0.35 \%$ across epochs. When compared with the fixation baseline condition, signal intensity increases in response to fearful faces occurred during the first two fear presentation epochs but were attenuated to baseline with subsequent stimulus presentations. In contrast, however, note that signal intensity decreases in response to happy faces persisted through all presentation epochs.

Figure $3 A$ depicts BOLD fMRI signal changes for the masked fear versus happy contrast in the most anterior coronal slice from the amygdaloid region of activation depicted in Figure 1. The ventral portion of this activation is within the temporal lobe, where the most anterior extent of the amygdala is located; however, this activation extends immediately rostral and dorsal to the traditionally defined amygdala within the region of the sublenticular substantia innominata (SI) of the basal forebrain (see Heimer et al., 1997). Within the SI this activation extends in a dorsomedial direction to the base of the anterior commissure.

Although BOLD signal changes in the ventral portion of this activation increased to fearful faces and decreased to happy faces (similar to the activation in Fig. 2), significant BOLD signal changes in the dorsal portion of this activation for the masked fear versus masked happy contrast $\left(p<6.6 \times 10^{-4}\right)$ were created by signal increases to both fearful and happy faces, in which increases to fearful faces were significantly larger. Figure $3 B$ presents an enlargement of the significant voxels of activation 

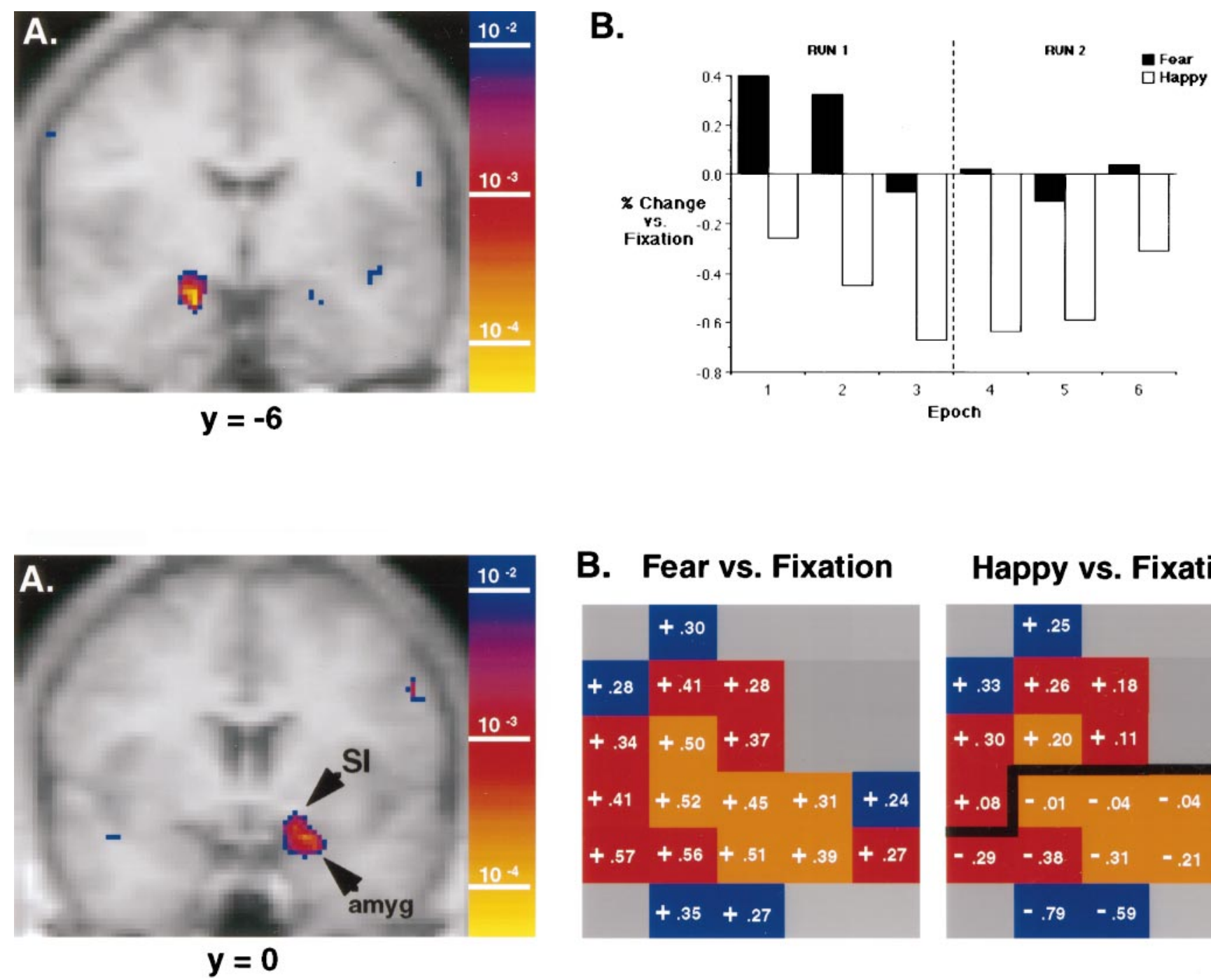

\section{B. Fear vs. Fixation}

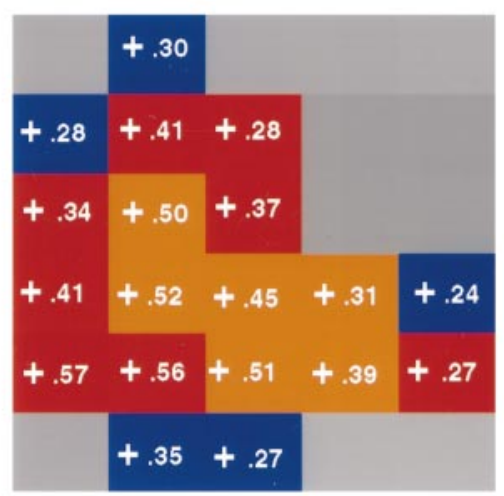

Happy vs. Fixation

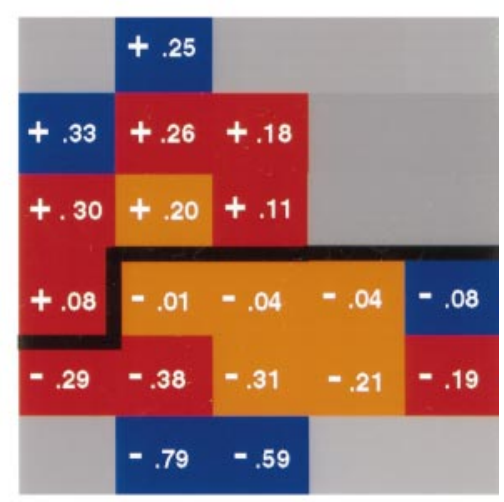

Figure 2. Top. Amygdala activation to masked fearful versus masked happy faces. $A$, Coronal display of the most posterior slice depicting activation within the region of the amygdala from Figure 1. Image parameters are as in Figure 1. Activation depicted here in the right amygdala includes four contiguous voxels that significantly increased in response to masked fearful faces when compared with masked happy faces $\left(p<6.6 \times 10^{-4}\right)$. B , Bar graph depicting changes in BOLD signal intensity as a function of repeated stimulus presentations. Bars represent the mean percentage change of signal intensity per epoch in response to masked fearful and masked happy faces (counterbalanced), as compared with the preceding and following low-level fixation conditions. Values reflect only the four voxels that exceeded the Bonferroni-corrected significance threshold (depicted within $A$ ) for the masked fearful versus masked happy contrast.

Figure 3. Bottom. Amygdala/SI activation to masked fearful versus masked happy faces. $A$, Coronal display of the most anterior slice depicting activation within the region of the amygdala from Figure 1. Image parameters are as in Figure 1. Note that the most ventral portion of this activation is within the temporal lobe (where the most anterior extent of the amygdala is located). This activation then extends dorsally into the basal forebrain where the sublenticular substantia innominata $(S I)$ is located. Six voxels met the Bonferroni-corrected significance threshold $\left(p<6.6 \times 10^{-4}\right)$. $B$, Enlargement of the activation presented in $A$ is presented twice: once for masked fear versus fixation and again for masked happy versus fixation. Colors of enlarged voxels represent significance levels for the original masked fear versus masked happy contrast as follows: orange, $p<6.6 \times 10^{-4} ;$ red, $p<0.005 ;$ blue, $p<0.05$. Although the significant statistical effect for the masked fear versus masked happy contrast in the ventral portion of this activation (amyg) is attributable to signal increases to fearful faces and signal decreases to happy faces (similar to the amygdala activation in Fig. 2), the statistical effect in the dorsal portion of this activation $(S I)$ is a result of signal increases to both fearful and happy faces in which increases to fearful faces are significantly larger $(p<6.6 \times$ $\left.10^{-4}\right)$.

from the masked fear versus masked happy contrast pictured in Figure $3 A$. The activation is presented twice: once for the fear versus fixation contrast and again for the happy versus fixation contrast. Numbers overlying the voxels present the average percentage of BOLD signal change from the fixation baseline across all stimulus presentations. Note that, in response to masked fear faces, all voxels of activation - the most ventral voxels located in the amygdala and the most dorsal voxels located in the SI- demonstrate signal increases. In contrast, in response to masked happy faces, ventrally located voxels demonstrate signal decreases (similar to the amygdala response depicted in Fig. 2), whereas dorsal voxels located in the SI demonstrate signal increases.

Table 1 presents Talairach coordinates (Talairach and Tournoux, 1988) and probability values of activated brain regions for the masked fear versus happy contrast for the eight subjects that reported not having seen the masked fearful and happy faces. 
Table 1. Talairach coordinates for masked fearful vs masked happy faces

\begin{tabular}{|c|c|c|c|c|c|}
\hline \multirow[b]{2}{*}{ No. } & \multirow[b]{2}{*}{ Location in brain } & \multirow[b]{2}{*}{$p$ value } & \multicolumn{3}{|c|}{ Talairach coordinates } \\
\hline & & & $x$ & $y$ & $z$ \\
\hline 1. & Amygdala & $3.7 \times 10^{-5}$ & 18 & -6 & -15 \\
\hline $2 \mathrm{a}$. & Amygdala/ & $1.6 \times 10^{-4}$ & -15 & 0 & -12 \\
\hline $2 b$. & Substantia innominata & $2.9 \times 10^{-4}$ & -12 & 0 & -9 \\
\hline 3. & Inferior prefrontal cortex & $1.6 \times 10^{-4}$ & -28 & 42 & 9 \\
\hline
\end{tabular}

Areas of significantly increased BOLD fMRI signal intensity across whole brain during presentation of masked fearful faces versus masked happy faces. Similar to Figures $1-3$, data presented are for the eight subjects who reported not having seen fearful or happy faces. Reported probability values and Talairach coordinates reflect the maximum activated voxel. Activation 1 corresponds to the activation pictured in Figure 2. Activation 2a, b corresponds to the activation pictured in Figure 3. The location of maximum voxels within the temporal lobe (amygdala) and basal forebrain (substantia innominata) are reported in $2 \mathrm{a}$ and $2 \mathrm{~b}$, respectively. The Talairach atlas (Talairach and Tournoux, 1988) suggests that the location of Activation 2a should be described as temporal cortex. Our designation of this locus as within the amygdala is based on (1) inspection of high resolution structural data, (2) other human structural atlases (DeArmond et al., 1993) depicting the amygdala as prominent at the anterior to posterior location of the anterior commissure $(y=0)$, and $(3)$ the similar response topography to the amygdala activation in Figure 2 (increase to fear, decrease to happy). Because activation of inferior frontal cortex was not predicted and our Bonferroni corrected significance threshold is based on the smaller size of the amygdala, we note that activation of this region did not achieve the appropriate Bonferroni corrected threshold for whole brain (see Results).

Activation within the left and right amygdala exceeded the a priori threshold for significant activation $\left(p<6.6 \times 10^{-4}\right)$. To obviate bias, we report here all areas of activation across whole brain that met this threshold. One other brain area, left inferior prefrontal cortex, also met this criterion (see Fig. 1, row one, slice nine). Because it was not predicted, we note then that activation in this region did not achieve the appropriate Bonferronicorrected threshold for whole brain $\left(p<1.0 \times 10^{-7}\right)$.

\section{Technical considerations}

Quantification of motion-Corrected motion during functional image acquisition was $<1.5 \mathrm{~mm}$ for all subjects. Missing data-A computer failure during image acquisition of subject number 7 caused his first run to be lost. This did not affect the results, because statistical effects were similar whether six, seven, or eight subjects were considered. The fact that the results were similar to those of six subjects is important, because loss of these data did not compromise the protection afforded by counterbalancing. Vessel effects-To rule out the possibility that the observed activations were attributable to flow through large vessels located medial to the temporal lobe (e.g., inferior carotid, middle cerebral artery), we acquired MR angiograms for these eight subjects, placed them into normalized Talairach space, and then averaged them across the subjects. Thus, the composite activations for eight subjects did not overlie the location of large vessels on averaged MR angiograms. Susceptibility artifact-It is unlikely that the observed activation is an artifact of changes in the nearby field of susceptibility because (1) inspection of animated raw signal intensity changes over time revealed no obvious systematic changes in susceptibility that mirrored counterbalanced conditions, and (2) these results (i.e., increased signal to fear, decreased signal to happy) are consistent with a previous positron emission tomography (PET) study using nonmasked stimuli, where susceptibility is not a concern.

\section{DISCUSSION}

\section{Significance of amygdala activation to masked stimuli}

Amygdala activation was observed in response to masked fearful faces versus masked happy target faces that subjects reported not having seen. As predicted, the backward masking of emotional facial expressions resulted in impressive isolation of the amygdala. This finding is particularly striking when considered in light of an earlier neuroimaging study demonstrating activation of the amygdala and four additional brain regions to presentation of nonmasked fearful faces versus happy faces (Morris et al., 1996).

These data highlight the automaticity of the amygdala response and are consistent with the assertion of LeDoux (1996) that the amygdala responds to early, crude representations of external stimuli. Although consistent with the notion that the amygdala might receive stimulus information directly from the thalamus (LeDoux et al., 1985), prior or parallel to elaborate cortical processing, the temporal resolution of the present design (based on $28 \mathrm{sec}$ epoch lengths) does not address this issue directly. In addition, portions of candidate cortical areas that also might survey masked facial stimuli [e.g., temporal visual cortex (Hasselmo et al., 1989) and ventral prefrontal cortex (Tranel et al., 1995; Hornak et al., 1996)] may not have been visualized in the present study because of characteristic signal drop-out associated with fMRI caused by the nearby sinus space. Replication of the present effect using PET (where susceptibility drop-out is not an issue) would address this concern.

\section{Direction of signal changes in the amygdala}

Within the amygdala, signal intensity increased to masked fear faces and decreased to masked happy faces, consistent with a previous imaging study (Morris et al., 1996). In addition, signal increases to masked fearful faces habituated. Habituation of response within the amygdala to emotionally valenced stimuli that have proven inconsequential is consistent with previous reports in both animals (Bordi et al., 1993) and humans (Breiter et al., 1996; Irwin et al., 1996). In contrast, however, signal intensity decreases in response to happy faces persisted through all presentation epochs. Happy faces appear to provide information of enduring importance and, in this sense, might be conceptualized as safety signals. One interpretation of these data is that both fearful and happy faces provide information about the potential for threat in a given environment, differentially affecting the level of activity in the amygdala.

This differential amygdala response, based on the valence of facial expressions, is consistent with a recent study of human infants in which presentation of negatively valenced (angry) faces produced increased eyeblink magnitudes and positively valenced (happy) faces produced decreased eyeblink magnitudes [Balaban (1995); see also Lang (1995)]. Activation of the amygdala is known to modulate eyeblink reflex sensitivity (Davis, 1992). To elaborate, although direct electrical stimulation of the amygdala 
in animals does not produce an eyeblink, the magnitude of the next eyeblink that is elicited after amygdala stimulation is modified (Whalen and Kapp, 1991). These converging results imply that, although amygdala activation to experimental presentation of emotionally valenced facial expressions may not produce obvious overt responses, it modifies overt responses to subsequent sensory information through numerous efferent pathways (see Kapp et al., 1992).

\section{Significance of SI activation}

The present experiment also produced activation that extended into the sublenticular SI of the basal forebrain, consistent with recent PET studies demonstrating blood flow changes within the SI that correlate with (1) recall of negatively valenced film stimuli (Cahill et al., 1996) as well as (2) thalamic responses to aversively conditioned stimuli (Morris et al., 1997). These data are also consistent with an emerging understanding of the inter-related anatomy of this region. For example, the "extended amygdala," including the bed nucleus of the stria terminalis (BNST; see Davis et al., 1997), describes a subset of neurons within the SI characterized as a functional and anatomical continuum of the traditionally defined amygdala, based on similarities in cytoarchitecture as well as neurotransmitter and projection systems (Heimer et al., 1997). In addition, the amygdala is known to project directly to the SI (Russchen et al., 1985) where the cholinergic nucleus basalis of Meynert (NBM) neurons are located (Mesulam et al., 1983).

The present demonstration of increased activation of the SI to both fearful and happy faces is consistent with (1) data in primates demonstrating increased neuronal responses in the SI to both positive (Richardson et al., 1988; Wilson and Rolls, 1990) and negative (Whalen et al., 1994) stimuli, (2) the well established role of the NBM in the modulation of cortical neuronal excitability (Celesia and Jasper, 1966; Szerb, 1967; Sillitto and Kemp, 1983; Metherate and Ashe, 1991), and (3) the fact that the activity of these neurons is more closely associated with an animal's overall state of vigilance or arousal rather than with its response to specific stimulus presentations (Detari and Vanderwolf, 1987; Whalen et al., 1994). Thus, activation of the SI to both fearful and happy facial expressions might represent a more generalized response to the salience (see Morris et al., 1997) or arousal value (see Kapp et al., 1992; Whalen et al., 1994) of these stimuli.

The dissociation between an amygdala response based on valence and an SI response based on arousal offers a possible neuroanatomical substrate for psychophysiological responses long hypothesized by Lang and colleagues to reflect emotional "action dispositions," based on the valence versus arousal value of presented stimuli (see Lang, 1995; Shupp et al., 1997). In addition, this response dissociation replicates both the earlier Morris et al. (1996) and Breiter et al. (1996) findings and clarifies response differences between the two studies. To elaborate, in the present study the amygdala demonstrated increases to fear and decreases to happy faces, similar to Morris et al. (1996); activations that extended into the SI demonstrated increases to both fear and happy faces, similar to Breiter et al. (1996).

\section{Explicit knowledge and the determination of awareness}

The present study was designed as a demonstration of the automaticity of amygdala response and as an initial effort toward determining whether awareness is a prerequisite for amygdala activation. Toward this end we used a stimulus onset asynchrony
(SOA) parameter $(33 \mathrm{msec})$ below a threshold previously determined to prevent awareness of masked emotional facial expressions (Esteves and Öhman, 1993). Still, numerous methodological differences between the present and previous studies preclude generalizations about awareness on the basis of the parameter of SOA alone.

There are several challenges associated with the measurement of awareness (see Merikle, 1992; Greenwald et al., 1996). These include the timing of measurement [during interstimulus intervals (ISIs) vs after completion of all stimulus presentations] and the type of measurement (objective vs subjective; recall vs recognition). For this initial study we chose to assess awareness subjectively after completion of all stimulus presentations, emphasizing recall versus recognition measures. The timing we chose does limit interpretation, to the extent that debriefing results may not accurately reflect the subjects' awareness at the time of presentation. However, this strategy (1) allowed for shorter ISIs, maximizing the number of masked facial expressions presented within each epoch and thereby protecting against type II error in terms of amygdala activation, and (2) obviated the need for subjects to be informed of the presence of masked stimuli before the study so that findings would not be confounded by explicit attempts to detect emotional expressions. Our reliance on recall versus recognition measures of explicit knowledge was guided by research demonstrating that recognition tasks requiring discrimination of very similar stimuli are more susceptible to incorrect identification of target items (Underwood, 1965; Roediger, 1980; Hintzman et al., 1992; Hintzman and Curran, 1995; Mäntylä, 1997). With specific reference to face stimuli, Mäntylä has demonstrated that recognition of faces is driven by familiarity processes versus detailed episodic information (Mäntylä, 1997).

An interstimulus objective forced choice task remains the "gold standard" for the definition of awareness in behavioral psychology (see Greenwald et al., 1996). Future studies using such an interstimulus measure would address the question of awareness directly but would change the nature of the task fundamentally (active search vs passive viewing). Use of an objective forced choice measure after completion of all stimulus presentations may enable the objective assessment of awareness during a passive viewing task, although this approach assumes that only consciously perceived information will influence objective responses (see Jacoby, 1991; Merikle, 1992; Seamon et al., 1995).

\section{Conclusions and future direction}

The role of the amygdala in fear conditioning is well established (Aggleton, 1992), and the more recent reports of its involvement in the processing of emotional facial expression (Adolphs et al., 1995; Breiter et al., 1996; Calder et al., 1996; Morris et al., 1996) are consistent with this role. The present study builds on the growing number of neuroimaging studies demonstrating that human amygdala activation in response to emotionally valenced stimuli is a reliable phenomenon (Breiter et al., 1996; Cahill et al., 1996; Irwin et al., 1996; Morris et al., 1996; Reiman et al., 1997). Specifically, we demonstrated isolated amygdala activation in response to masked presentations of facial expressions that prevented explicit knowledge. These data underscore the automaticity (see McNally, 1995) of the processing of the emotional facial expressions of the amygdala and are consistent with data implicating the amygdala in the nonconscious monitoring of emotional stimuli (Halgren, 1992; Öhman, 1992; LeDoux, 1996).

In light of the present findings, we note that subjects with anxiety disorders demonstrate (1) amygdala activation in associ- 
ation with symptoms (see Rauch and Shin, 1997) and (2) information-processing biases during nonconscious processing (see Öhman, 1992; Mathews and MacLeod, 1994; Mogg et al., 1995; McNally et al., 1996). We are developing fMRI probes, using masked stimulus presentations, in an attempt to elucidate the role of the amygdala in the clinical phenomena observed across the anxiety disorders (e.g., hypervigilance, failure to habituate, exaggerated startle, etc.).

\section{REFERENCES}

Adolphs R, Tranel D, Damasio H, Damasio A (1995) Fear and the human amygdala. J Neurosci 15:5879-5891.

Aggleton JP, editor (1992) The amygdala: neurobiological aspects of emotion, memory, and mental dysfunction. New York: Wiley.

Balaban MT (1995) Affective influences on startle in five month old infants: reactions to facial expressions of emotion. Child Dev 66:28-36.

Berns GS, Cohen JD, Mintun MA (1997) Brain regions responsive to novelty in the absence of awareness. Science 276:1272-1275.

Bordi F, LeDoux JE, Clugnet MC, Pavlides C (1993) Single-unit activity in the lateral nucleus of the amygdala and overlying areas of striatum in freely behaving rats: rates, discharge patterns, and responses to acoustic stimuli. Behav Neurosci 107:757-769.

Breiter HC, Etcoff NL, Whalen PJ, Kennedy WA, Rauch SL, Buckner RL, Strauss MM, Hyman SE, Rosen BR (1996) Response and habituation of the human amygdala during visual processing of facial expression. Neuron 17:875-887.

Cahill L, Haier RJ, Fallon J, Alkire MT, Tang C, Keator D, Wu J, McGaugh JL (1996) Amygdala activity at encoding correlated with long-term, free recall of emotional information. Proc Natl Acad Sci USA 93:8016-8021.

Calder AJ, Young AW, Rowland D, Perrett DI, Hodges JR, Etcoff NL (1996) Facial emotion recognition after bilateral amygdala damage: differentially severe impairment of fear. Cognit Neuropsychol 13:699-745.

Celesia GG, Jasper HH (1966) Acetylcholine released from cerebral cortex in relation to state of activation. Neurology 16:1053-1064.

Cohen MS, Weisskoff RM (1991) Ultra-fast imaging. Magn Reson Imaging 9:1-37.

Davis M (1992) The role of the amygdala in conditioned fear. In: The amygdala: neurobiological aspects of emotion, memory, and mental dysfunction (Aggleton JP, ed), pp 255-306. New York: Wiley.

Davis M, Walker DL, Lee Y (1997) Roles of the amygdala and bed nucleus of the stria terminalis in fear and anxiety measured with the acoustic startle reflex. In: Psychobiology of posttraumatic stress disorder (Yehuda R, McFarlane AC, eds), pp 305-331. New York: New York Academy of Sciences.

DeArmond SJ, Fusco MM, Dewey MM (1993) Structure of the human brain, 3rd Ed, pp 140-141. New York: Oxford UP.

Detari L, Vanderwolf CH (1987) Activity of identified cortically projecting and other basal forebrain neurones during large slow waves and cortical activation in anesthetized rats. Brain Res 437:1-8.

Ekman P, Friesen WV (1976) Pictures of facial affect. Palo Alto, CA: Consulting Psychologists.

Esteves F, Öhman A (1993) Masking the face: recognition of emotional facial expressions as a function of the parameters of backward masking. Scand J Psychol 34:1-18.

Filipek P, Richelme C, Kennedy D, Caviness V (1994) The young adult human brain: an MRI-based morphometric analysis. Cereb Cortex 4:344-360.

Greenwald AG, Draine SC, Abrams RL (1996) Three cognitive markers of unconscious semantic activation. Science 273:1699-1702.

Halgren E (1992) Emotional neurophysiology of the human amygdala within the context of human cognition. In: The amygdala: neurobiological aspects of emotion, memory, and mental dysfunction (Aggleton JP, ed), pp 191-228. New York: Wiley.

Hasselmo ME, Rolls ET, Baylis GC (1989) The role of expression and identity in the face-selective responses of neurons in the temporal visual cortex of the monkey. Behav Brain Res 32:203-218.

He S, Cavanaugh P, Intrilligator J (1996) Attentional resolution and locus of visual awareness. Nature 383:334-337.

Heimer L, Harlan RE, Alheid GF, Garcia MM, De Olmos J (1997) Substantia innominata: a notion which impedes clinical-anatomical correlations in neuropsychiatric disorders. Neuroscience 76:957-1006.
Hintzman DL, Curran T (1995) When encoding fails: instructions, feedback, and registration without learning. Mem Cognit 23:213-226.

Hintzman DL, Curran T, Oppy B (1992) Effects of similarity and repetition on memory: registration without learning? J Exp Psychol 18:667-680.

Hornak J, Rolls ET, Wade D (1996) Face and voice expression identification in patients with emotional and behavioural changes following ventral frontal lobe damage. Neuropsychologia 34:247-261.

Irwin W, Davidson RJ, Lowe MJ, Mock BJ, Sorenson JA, Turski PA (1996) Human amygdala activation detected with echo-planar functional magnetic resonance imaging. NeuroReport 7:1765-1769.

Jacoby LL (1991) A process dissociation framework: separating automatic from intentional uses of memory. J Mem Language 30:513-541.

Jiang A, Kennedy DN, Baker JR, Weiskoff RM, Tootell RBH, Woods RP, Benson RR, Kwong KK, Brady TJ, Rosen BR, Belliveau JW (1995) Motion detection and correction in functional MR imaging. Hum Brain Mapp 3:224-235.

Kapp BS, Whalen PJ, Supple WF, Pascoe J (1992) Amygdaloid contributions to conditioned arousal and sensory information processing. In: The amygdala: neurobiological aspects of emotion, memory, and mental dysfunction (Aggleton JP, ed), pp 229-254. New York: Wiley.

Kwong KK (1995) Functional magnetic resonance imaging with echo planar imaging. Magn Reson Q 11:1-20.

Kwong KK, Belliveau JW, Chesler DA, Goldberg IE, Weisskoff RM, Poncelet BP, Kennedy DN, Hoppel BE, Cohen MS, Turner R, Cheng HM, Brady TJ, Rosen BR (1992) Dynamic magnetic resonance imaging of human brain activity during primary sensory stimulation. Proc Natl Acad Sci USA 89:5675-5679.

Lang PJ (1995) The emotion probe: studies of motivation and attention. Am Psychologist 50:372-385.

LeDoux JE (1996) The emotional brain. New York: Simon and Shuster.

LeDoux JE, Ruggiero DA, Reis DJ (1985) Projections to the subcortical forebrain from anatomically defined regions of the medial geniculate body in the rat. J Comp Neurol 242:182-213.

Mansfield P (1977) Multi-planar image formation using NMR spin echoes. J Physics [Suppl C] 10:L55-L58.

Mäntylä T (1997) Recollection of faces: remembering differences and knowing similarities. J Exp Psychol 23:1203-1216.

Mathews A, MacLeod C (1994) Cognitive approaches to emotion and emotional disorders. Annu Rev Psychol 45:25-50.

McNally RJ (1995) Automaticity and the anxiety disorders. Behav Res Ther 33:747-754.

McNally RJ, Amir N, Lipke HJ (1996) Subliminal processing of threat cues in posttraumatic stress disorder? J Anxiety Disord 10:115-128.

Merikle PM (1992) Perception without awareness: critical issues. Am Psychologist 47:792-796.

Mesulam M-M, Mufson EI, Wainer BH, Levey AI (1983) Central cholinergic pathways in the rat: an overview based on an alternative nomenclature (Ch1-Ch6). Neuroscience 10:1185-1201.

Metherate R, Ashe JH (1991) Basal forebrain stimulation modifies auditory cortex responsiveness by an action at muscarinic receptors. Brain Res 559:163-167.

Mogg K, Bradley BP, Williams R (1995) Attentional bias in anxiety and depression: the role of awareness. Br J Clin Psychol 34:17-36.

Morris JS, Frith CD, Perrett DI, Rowland D, Young AW, Calder AJ, Dolan RJ (1996) A differential neural response in the human amygdala to fearful and happy facial expressions. Nature 383:812-815.

Morris JS, Friston KJ, Dolan RJ (1997) Neural responses to salient visual stimuli. Proc R Soc Lond [Biol] 264:769-775.

Ogawa S, Tank DW, Menon R, Ellermann JM, Kim SG, Merkle H, Ugurbil K (1992) Intrinsic signal changes accompanying sensory stimulation: functional brain mapping using MRI. Proc Natl Acad Sci USA 89:5951-5955.

Öhman A (1992) Fear and anxiety as emotional phenomena: clinical phenomenology, evolutionary perspectives, and information-processing mechanisms. In: Handbook of emotions (Lewis M, Haviland JM, eds), pp 511-536. New York: Guilford.

Oldfield RC (1971) The assessment and analysis of handedness: the Edinburgh inventory. Neuropsychologia 9:97-113.

Rauch SL, Shin LM (1997) Functional neuroimaging studies in posttraumatic stress disorder. In: Psychobiology of posttraumatic stress disorder (Yehuda R, McFarlane AC, eds), pp 83-98. New York: New York Academy of Sciences.

Rauch SL, Whalen PJ, Savage CR, Curran T, Kendrick A, Brown HD, Bush G, Breiter HC, Rosen BR (1997) Striatal recruitment during an 
implicit sequence learning task as measured by functional magnetic resonance imaging. Hum Brain Mapp 5:124-132.

Reese TG, Davis TL, Weisskoff RM (1995) Automated shimming at 1.5 $\mathrm{T}$ using echo-planar image frequency maps. J Magn Reson Imaging 5:739-745.

Reiman EM, Lane RD, Ahern GL, Schwartz GE, Davidson RJ, Friston KJ, Yun L, Chen K (1997) Neuroanatomical correlates of externally and internally generated human emotion. Am J Psychiatry 154:918-925.

Richardson RT, Mitchell SJ, Baker FH, DeLong MR (1988) Responses of nucleus basalis of Meynert neurons in behaving monkeys. In: Cellular mechanisms of conditioning and behavioral plasticity (Woody CD Alkon DL, McGaugh JL, eds), pp 161-173. New York: Plenum.

Roediger HL (1980) Memory metaphors in cognitive psychology. Mem Cognit 8:231-246.

Rolls ET, Tovee MJ (1994) Processing speed in the cerebral cortex and the neurophysiology of visual masking. Proc R Soc Lond [Biol] 257:9-15.

Russchen FT, Amaral DG, Price JL (1985) The afferent connections of the substantia innominata in the monkey, Macaca fascicularis. J Comp Neurol 242:1-27.

Schacter DS, Chiu P, Ochsner KN (1993) Implicit memory: a selective review. Annu Rev Neurosci 16:159-182.

Seamon JG, Williams PC, Crowley MJ, Kim IJ, Langer SA, Orne PJ, Wishengrad DL (1995) The mere exposure effect is based on implicit memory: effects of stimulus type, encoding conditions and number of exposures on recognition and affect judgments. J Exp Psychol 21:711-721.
Shupp HT, Cuthbert BN, Bradley MM, Birbaumer N, Lang P (1997) Probe P3 and blinks: two measures of affective startle modulation. Psychophysiology 34:1-6.

Sillitto AM, Kemp JA (1983) Cholinergic modulation of the functional organization of the cat visual cortex. Brain Res 289:143-155.

Szerb JC (1967) Cortical acetylcholine release and electroencephalographic arousal. J Physiol (Lond) 192:329-345.

Talairach J, Tournoux P (1988) Co-planar stereotaxic atlas of the human brain. New York: Theime.

Tranel D, Damasio H, Damasio AR (1995) Double dissociation between overt and covert face recognition. J Cognit Neurosci 7:425-432.

Underwood BJ (1965) False recognition produced by implicit verbal responses. J Exp Psychol 70:122-129.

Whalen PJ, Kapp BS (1991) Contributions of the amygdaloid central nucleus to the modulation of the nictitating membrane reflex in the rabbit. Behav Neurosci 104:141-153.

Whalen PJ, Kapp BS, Pascoe J (1994) Neuronal activity within the nucleus basalis and conditioned neocortical electroencephalographic activation. J Neurosci 14:1623-1633.

Wilson FAW, Rolls ET (1990) Neuronal responses related to reinforcement in the primate basal forebrain. Exp Brain Res 509:213-231.

Woods RP, Cherry SR, Mazziotta JC (1992) Automated algorithm for aligning tomographic images. II. Cross-modality MRI-PET registration. J Comput Assist Tomogr 16:620-633.

Zajonc RB (1980) Feeling and thinking: preferences need no inferences. Am Psychologist 35:151-175. 